\title{
ERRATUM
}

Juan Cuadros · Ana Mazón · Rocío Martinez

Pilar González · Alberto Gil-Setas · Uxua Flores

Beatriz Orden · Peña Gómez-Herruz $\cdot$ Rosario Millan

\section{The aetiology of paediatric inflammatory vulvovaginitis}

Published online: 14 February 2004

(C) Springer-Verlag 2004

\section{Eur J Pediatr (2003) 163:105-107}

Due to an unfortunate error the column of isolates in cases was missing in Table 1. The correct table is given here.
Table 1 Microorganisms $(n=86)$ isolated from cases $(n=74)$ and controls $(n=11)$

\begin{tabular}{llll}
\hline Microorganism & Cases & Controls & $P$ \\
\hline Streptococcus pyogenes & 47 & 0 & $<0.001$ \\
Haemophilus influenzae & 12 & 0 & \\
Corynebacterium spp. & 8 & 2 & \\
Staphylococcus aureus & 4 & 0 & \\
Candida albicans & 3 & 0 & \\
Gardnerella vaginalis & 2 & 2 & \\
Escherichia coli & 2 & 1 & \\
Proteus mirabilis & 2 & 0 & \\
Staphylococcus epidermidis & 2 & 8 & \\
Candida parapsilosis & 1 & 0 & \\
Streptococcus pneumoniae & 1 & 0 & \\
Streptococcus anginosus & 1 & 3 & \\
Haemophilus parainfluenzae & 1 & 0 & \\
Pseudomonas aeruginosa & 0 & 1 & \\
\end{tabular}

The online version of the original article can be found at http://dx. doi.org/10.1007/s00431-003-1373-x

J. Cuadros $(\bowtie) \cdot$ P. Gómez-Herruz

Servicio de Microbiología y Parasitología,

Cra. de Meco s $/ n$,

Alcalá de Henares, 28805 Madrid,

Spain

E-mail: jcuadros@efd.net

Fax: + 34-1-8801825

A. Mazón · A. Gil-Setas

Laboratorio de Microbiología,

Ambulatorio General Solchaga,

Pamplona, Navarra, Spain

R. Martinez $\cdot$ B. Orden $\cdot$ R. Millan

Laboratorio de Microbiología,

CE Argüelles, Madrid, Spain

P. González

Servicio de Pediatría, Hospital Príncipe de Asturias,

Alcalá de Henares, Madrid, Spain

U. Flores

Servicio de Pediatría, Centro de Salud de Noain,

Navarra, Spain 